March 18-20, 2008, Faculty of Engineering, Tanta Univ., Egypt

\title{
Rapid Cardiac MRI Using Random Radial Trajectories
}

\author{
Shereen M. El-Metwally ${ }^{1 *}$, Khaled Z. Abd-Elmoniem² ${ }^{2}$ Abou-Bakr M. Youssef ${ }^{1}$, Yasser M. Kadah ${ }^{1}$ \\ ${ }^{1}$ Department of Systems and Biomedical Engineering, Cairo University, Cairo, Egypt \\ ${ }^{2}$ Department of Electrical and Computer Engineering, John Hopkins University, USA \\ *e-mail: shereen .elmetwally@k-space.org
}

\begin{abstract}
We introduce using Sparse MRI with random radial trajectories in order to allow MRI images reconstruction from a small number of acquired $k$-space data, so fulfilling the demands of dynamic MRI. Different random radial trajectories are generated by varying the parameters of the added random perturbations to the radial trajectories. Images reconstruction is performed using the non-linear L1 norm reconstruction. Entropy is computed for the reconstructed images, as a quantitative measure for the reconstructed image quality. Both phantom simulation and real cardiac images are used in our experiments. Our results show that more sparsely sampled images can be reconstructed with higher quality compared to using nonrandomly sampled radial $k$-space trajectories.
\end{abstract}

Keywords: Cardiac Magnetic Resonance Imaging (MRI), radial k-space sampling, dynamic imaging

\section{INTRODUCTION}

Patient motion during MRI cardiac imaging causes artifacts in the reconstructed image that obscure anatomical details. The main sources of these artifacts are cardiac and respiratory motion. Dynamic MRI captures an object in motion by acquiring a series of images at a high frame rate, thereby reducing motion artifacts. Dynamic imaging places conflicting demands requiring both high spatial resolution to resolve anatomical detail, and high temporal resolution to monitor rapid changes in signal. However, $k$-space sampling that obeys the Nyquist theorem usually precludes simultaneous achievement of both aims. $k$-space undersampling speeds up the acquisition by only sampling part of the required $k$-space. Sparse MRI is a fast imaging method based on undersampled $k$-space sampling and non-linear reconstruction [1]. This approach is inspired by theoretical results in sparse signal recovery $[2,3]$ showing that if the underlying image is compressible it can be recovered from randomly undersampled frequency data, an idea known as compressed sensing. It exploits the fact that medical images often have a sparse representation in some domain (such as finite differences, wavelets, Fourier, etc.), where the number of coefficients needed to describe the image accurately is significantly smaller than the number of pixels in the image. Uniform undersampling of the Fourier domain results in aliasing. When the undersampling is random, the aliasing is incoherent acting as additional noise interference in the image representing incoherent interference of the sparse transform coefficients. Sparsity is exploited by constraining the reconstruction to have a sparse representation and to be consistent with the measured $k$-space data. The success of the reconstruction depends on the sparsity of the coefficients and that the interference is small, having random statistics. This approach has been used with randomly perturbed undersampled spirals [4] and with randomly undersampled 3DFT trajectories [5]. It has been shown that the used non-linear L1 norm reconstruction outperformed conventional linear reconstruction, recovering the image even with severe undersampling.

Radial trajectories have many favorable intrinsic properties with respect to the demands of dynamic MRI including [6]:

1) Motion-induced artifacts result predominantly in radial streaks with only low intensity near the source of motion and reduce motion-induced ghosting. No ghosts displaced along phaseencoding direction are present. 


\section{$25^{\text {th }}$ NATIONAL RADIO SCIENCE CONFERENCE (NRSC 2008)}

\section{$2 \quad \mathrm{~K} 01$}

\section{March 18-20, 2008, Faculty of Engineering, Tanta Univ., Egypt}

2) The coverage of the $k$-space center in each radial line avoids contrast continuities and preserves the continuity of the process. Also, oversampling of the low spatial frequencies provides intrinsic averaging of the gross features of the subject.

3) By applying a magnitude reconstruction, a reduced sensitivity to statistical phase errors may be achieved, although the amplitude of motion-induced artifacts increases.

Random sampling may not be feasible in MR as the $k$-space trajectories have to be smooth due to hardware and physiologic considerations. Radial trajectories besides being fast and time-efficient, they result in $k$-space sampling with a variable density increasing linearly with the inverse of the distance from the $k$-space origin. Therefore, they are a good candidate to approximate random sampling due to although they span $k$-space uniformly; they are far from being regular as in Cartesian grid sampling.

In this paper, we introduce using Sparse MRI with random radial trajectories to exploit the intrinsic advantages of these trajectories with respect to the demands of dynamic MRI. Different random radial trajectories are generated by varying the parameters of the added random perturbations to the radial trajectories. Images reconstruction is performed using the non-linear L1 norm reconstruction. Entropy is then computed for the reconstructed images, as a quantitative measure for the reconstructed image quality. Our results show that more sparsely sampled images can be reconstructed with higher quality compared to using uniformly sampled radial $k$ space trajectories.

\section{METHODOLOGY}

The block diagram in Figure 1 summarizes the main applied steps in our work. This section describes these steps in details.

\section{(A)Random Radial Perturbations}

Radial lines are perturbed by adding slight random deviations taken from Guassian distribution with zero mean and varying variances. Different schemes for the random perturbations are used in the simulations. These include 1) using constant variance along radial lines, and 2) using montonically increasing variance with the distance from the $k$-space origin. i.e., variance is increased in areas of low sampling density and decreased in areas of high sampling density.

Due to the impracticality of pure random sampling of $k$-space, a practical incoherent sampling scheme is aimed to closely mimic the interference properties of pure random undersampling. Therefore, the simulated random radial trajectories are processed using a numerical algorithm [7] to keep the applied RF gradients amplitude and slew rate below the maximum permissible limits.

\section{(B) Gridding Reconstruction}

In MRI, gridding has been used routinely with respect to nonuniform, non-Cartesian sampling of the $k$-space. Conventional gridding is applied here to compare with non-linear L1 norm reconstruction using the randomly perturbed radial trajectories. The gridding algorithm is basically performed in four steps [8]:

1) Precompensate the data with inverse of the sampling density to compensate for the varying density of sampling in $k$-space.

2) Convolve with a Kaiser-Bessel window and resample onto a Cartesian grid.

3) Fourier transform.

4) Postcompensate to remove the apodization of the convolution kernel by dividing by the transform of the Kaiser-Bessel window.

\section{(C)Non-linear L1 norm Conjugate- Gradient Reconstruction}

Image reconstruction is performed by solving the following constrained optimization problem [1]: 
March 18-20, 2008, Faculty of Engineering, Tanta Univ., Egypt

$\min \lambda\|\Psi(f)\|_{1}+\alpha T V(f)$
s.t. $\|N F F T(f)-y\|_{2}<\varepsilon$

where $f$ is the reconstructed image, $y$ is the measured $k$-space data, $\Psi$ is the sparsifying transform operator, TV or Total-variation is the finite-differences sparsifying transform. Minimizing the objective function promotes sparsity by both the specific transform and finite-differences at the same time. NFFT stands for the nonuniform fast Fourier transform of the image. $\varepsilon$ controls the fidelity of the reconstruction to the measured data. The threshold parameter $\varepsilon$ is usually set below the expected noise level. $\alpha$ trades $\Psi$ sparsity with finite-differences sparsity. $\lambda$ is a regularization parameter that determines the trade-off between the data consistency and sparsity. An iterative non-linear conjugate gradient descent algorithm with backtracking line search is used, following the work in [1]. In our work, image reconstruction is done in the sparse spatial image domain, i.e., no sparifying transform is used.

\section{(D) Entropy Minimization}

The entropy criterion, E, is defined as [9]:

$$
E=-\sum_{j=1}^{N} \frac{B_{j}}{B_{\max }} \ln \left[\frac{B_{j}}{B_{\max }}\right]
$$

where $N$ is the number of image pixels and $B_{j}$ is the modulus of the complex value of the $j$ th image pixel or the pixel brightness. $B_{\max }$ is given by

$$
B_{\max }=\sqrt{\sum_{j=1}^{N} B_{j}^{2}}
$$

When all the image energy is located in a single pixel and the remaining pixels are black. When a $128 \mathrm{x} 128$ image has a uniform brightness, $B_{j} / B_{\max }=1 / 128$ for all the pixels and the entropy $\mathrm{E}=621$. Therefore, entropy minimization favors high contrast. This entropy criterion favors alterations to the data that tend to increase the number of dark pixels. It has been used before as a focus criterion to remove motion-induced ghosts from low intensity regions of the image that would otherwise be dark. Entropy minimization is used here to as a measure of the reconstructed image quality.

\section{EXPERIMENTAL VERIFICATION}

\section{(A)Phantom simulation}

A 2D numerical SheppLogan phantom is used. The phantom image is designed as a linear superposition of elliptical objects, whose FTs are scaled "jinc" functions (jinc $\mathrm{x}=J_{1}(\mathrm{x}) /(2 \mathrm{x})$, where $J_{1}$ is a first-order Bessel function. $k$-space samples can thus be evaluated directly, therefore the phantom simulates realistic $k$-space sampling and truncation.

Phantom reconstruction is done using both non-linear conjugate-gradient method and the conventional gridding reconstruction. The reconstructed phantom resolution is $160 \times 160$. $k$-space undersampling with 8 -fold is used. That is, $k$-space consists of 20 radial lines. Each line consists of 512 samples. Practically, each radial line is acquired during TR interval, which is relatively long. Therefore, the number of samples per line can be increased, as much as permitted by TR, without any increase in the overall acquisition time.

\section{(B)MRI data}

Cardiac MRI magnitude images are Fourier transformed at various generated random radial trajectories using non-uniform Fourier transform. Undersampling factors of 8 and 10 are used. $k$-space consists of 16 radial lines 


\title{
$25^{\text {th }}$ NATIONAL RADIO SCIENCE CONFERENCE (NRSC 2008)
}

\author{
March 18-20, 2008, Faculty of Engineering, Tanta Univ., Egypt
}

with 512 samples/line in the case of 8-fold acceleration, and consists of 13 radial lines with 512 samples/line in the case of 10-fold acceleration. The reconstructed image resolution is $128 \times 128$.

\section{RESULTS AND DISCUSSION}

The results of simulation phantom reconstruction with 8-fold acceleration are shown in Figure 2. It can be seen that the small ellipses, as shown surrounded by the dashed rectangle, become more resolved with increasing the variance of the added random deviations. This is also accompanied by an overall decrease in image blurring. Image reconstructed using conventional gridding shows much lower quality, compared to those obtained using non-linear reconstruction. Table 1 demonstrates the computed entropy of the reconstructed phantom at the different variances used. It can be noticed that entropy value decreases with added variance increase. Linearlyincreasing variance shows entropy with slightly higher values compared to non-changing variance.

Figures 3 and 4 display cardiac image reconstruction using 8-fold and 10-fold accelerations respectively. It can be seen that the fine image details become clearer with added random perturbations compared to using nonrandom radial trajectory. Also, as variance increases, more contrast enhancement is noticed. Even at very sparse undersampling of 10 -fold, image reconstruction revealed more improvement. Table 2 demonstrates the computed entropy of the reconstructed images using non-linear reconstruction with random radial perturbations at different variances. It can be seen from the table that entropy decreases with variance increasing. Images reconstructed using conventional gridding method show lower quality compared to non-linear reconstruction.

\section{CONCLUSIONS}

Using randomly perturbed radial $k$-space enables more sparsely sampled image reconstruction with higher quality compared to using non-randomly sampled radial $k$-space trajectories. Future research should include the investigation of using sparsity transform in non-linear reconstruction of radially sampled images. Also, a study of varying the non-linear reconstruction parameters is to be done for different image models such as piece-wise varying and smoothly varying models.

\section{REFERENCES}

[1] M. Lustig, D. Donoho and J. Pauly, "Sparse MRI: The application of compressed sensing for rapid MR imaging", Magnetic Resonance in Medicine, Vol. 58, Issue 6, pp. 1182-1195, December 2007.

[2] E. Candès, J. Romberg and T. Tao "Robust uncertainty principles: Exact signal reconstruction from highly incomplete frequency information”, IEEE Transactions on Information Theory, Vol. 52, No. 2, pp. 489-509, February 2006.

[3] D. Donoho, "Compressed sensing", IEEE Transactions on Information Theory, Vol. 52, No. 4, pp. 1289-1306, April 2006.

[4] M. Lustig, J. Lee, D. Donoho and J. Pauly, "Faster imaging with randomly perturbed undersampled spirals and L1 reconstruction", Proceedings of the ISMRM, Miami Beach, Florida, USA, May 2005.

[5] M. Lustig, D. Donoho and J. Pauly, "Rapid MR imaging with compressed sensing and randomly undersampled 3DFT trajectories", Proceedings of the ISMRM, Seattle, Washington, USA, May 2006.

[6] V. Rasche, R. de Boer, D. Holz and R. Proksa, "Continuous radial data acquisition for dynamic MRI", Magnetic Resonance in Medicine, Vol. 34, pp. 754-761, 1995.

[7] C. Hardy and H. Cline, "Broadband nuclear magnetic resonance pulses with two-dimensional spatial selectivity", Journal of Applied Physics, Vol. 66, No. 4, pp. 1513-1516, August 1989.

[8] J. Jackson, C. Meyer, D. Nishimura and A. Macovski, "Selection of a convolution function for Fourier inversion using gridding", IEEE Transactions on Medical Imaging, Vol. 10, No. 3, pp. 473-478, September 1991.

[9] D. Atkinson, D. Hill, P. Stoyle, P. Summers and S. Keevil, "Automatic correction of motion artifacts in magnetic resonance images using an entropy focus criterion”, IEEE Transactions on Medical imaging, Vol. 16, No. 2, pp. 903-910, December 1997. 
March 18-20, 2008, Faculty of Engineering, Tanta Univ., Egypt

TABLE 1

Entropy computed for phantom simulation with 8-fold acceleration.

\begin{tabular}{|c|c|c|}
\hline variance & 2. & Entropy \\
\hline 3. & 4. & 186.9193 \\
\hline 0.5 & 6. & 174.1913 \\
\hline 7. & 8. & 167.6517 \\
\hline 9. & 10. & 163.4419 \\
\hline $\begin{array}{l}11 . \quad \text { Linearly } \\
\text { increasing in the } \\
\text { range[0.5-2], slope=0.5 }\end{array}$ & 12. & 168.5984 \\
\hline $\begin{array}{l}14 . \quad \text { Linearly } \\
\text { increasing in the } \\
\text { range[0.5-2.75], } \\
\text { slope }=0.75\end{array}$ & 15. & 164.3755 \\
\hline
\end{tabular}

TABLE 2

Entropy computed for cardiac image reconstruction with 8-fold and 10-fold accelerations.

\begin{tabular}{|c|c|c|c|c|c|}
\hline 17. & variance & & $\begin{array}{l}18 . \\
\text { 8-fold } \\
\text { eration }\end{array}$ & $\begin{array}{r}\text { Entrop } \\
20 \\
a\end{array}$ & $\begin{array}{l}10 \text {-fold } \\
\text { eration }\end{array}$ \\
\hline 21. & 0 & 22. & 184.4959 & 23. & 188.1973 \\
\hline 24. & 0.5 & 25. & 182.0886 & 26. & 186.4292 \\
\hline 27. & 1 & 28. & 181.0794 & 29. & 185.6843 \\
\hline 30. & 2 & 31. & 179.4062 & 32. & 184.1696 \\
\hline 33. & 3 & 34. & 178.2825 & 35. & 182.9701 \\
\hline
\end{tabular}

Random radial perturbations with different variances

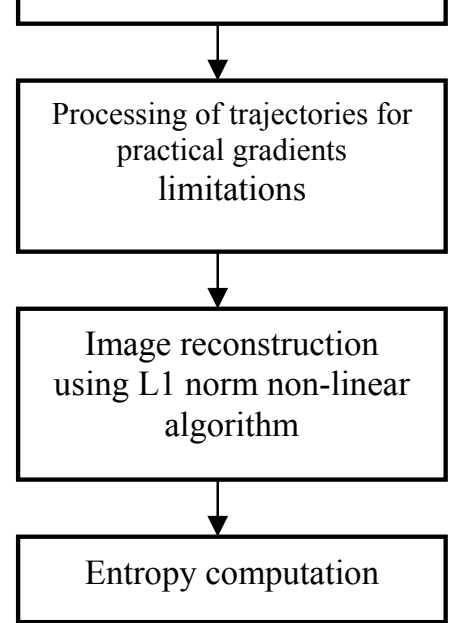

Figure 1. Block diagram for the main steps employed. 


\section{$25^{\text {th }}$ NATIONAL RADIO SCIENCE CONFERENCE (NRSC 2008)}

\section{\begin{tabular}{l|l|}
6 & K01 \\
\hline
\end{tabular}}

March 18-20, 2008, Faculty of Engineering, Tanta Univ., Egypt

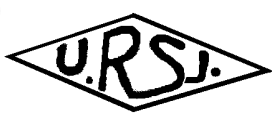

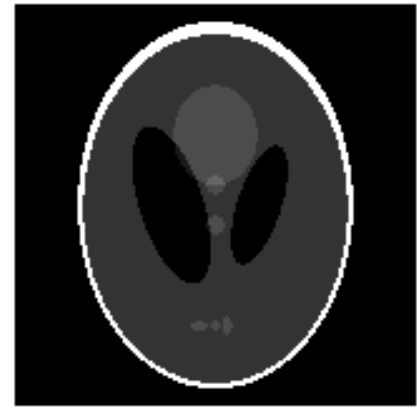

(a)

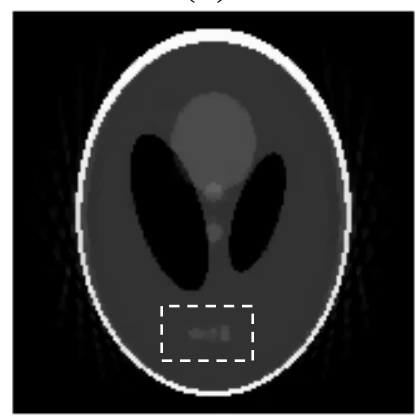

(d)

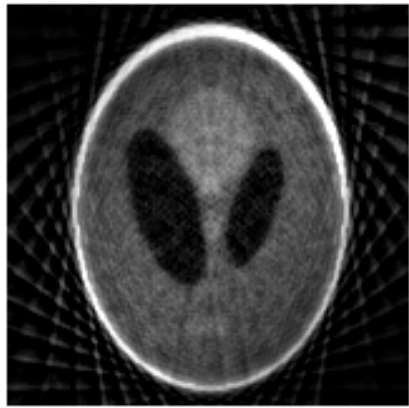

(b)

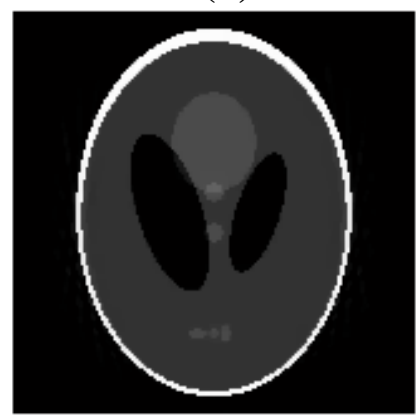

(e)

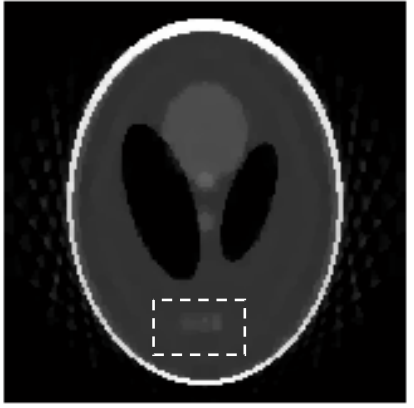

(c)

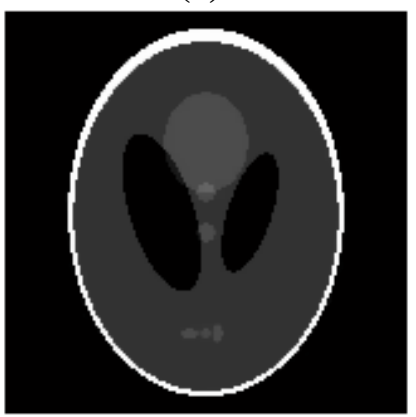

(f)

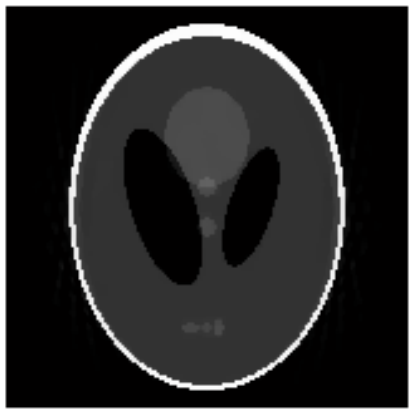

(g)

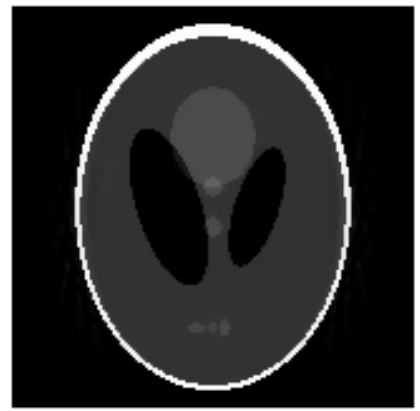

(h)

Figure 2. Phantom image reconstruction with 8-fold acceleration. (a) Original image. (b) Image reconstructed using conventional gridding. Non-linear conjugate gradient reconstruction using (c) non-random radial $k$ - space, random radial $k$-space with (d) variance $=0.5,(\mathrm{e})$ variance $=1$, (f) variance $=2,(\mathrm{~g})$ linearly increasing variance in the range $[0.5,2], \mathrm{h})$ linearly increasing variance in the range $[0.5,2.75]$. The dashed rectangle surrounds the smallest ellipses which exhibit a better resolution with variance increasing. 


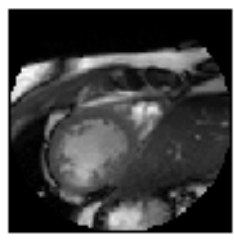

(a)

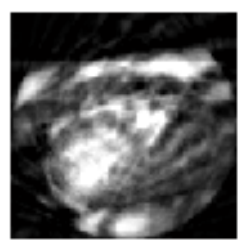

(b)

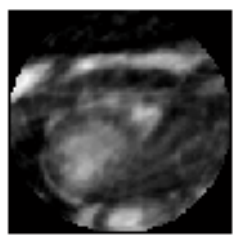

(c)

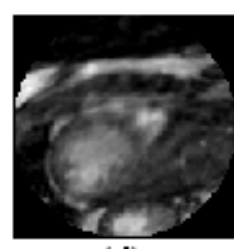

(d)

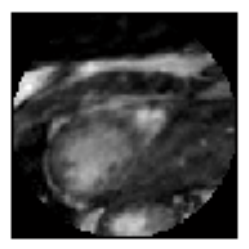

(e)

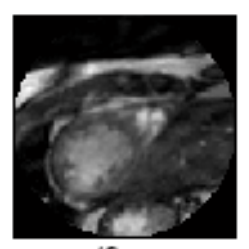

(f)

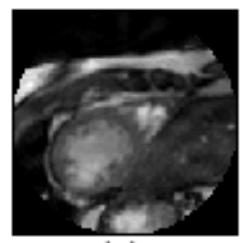

(g)

Figure 3. Cardiac image reconstruction with 8-fold acceleration. (a) Original image. (b) Image reconstructed using conventional gridding. Non-linear conjugate gradient reconstruction using (c) non-random radial $k$-space, random radial $k$-space with $(\mathrm{d})$ variance $=0.5,(\mathrm{e})$ variance $=1,(\mathrm{f})$ variance $=2,(\mathrm{~g})$ variance $=3$.

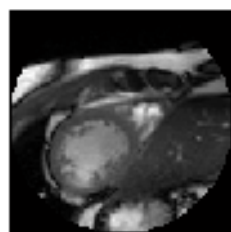

(a)

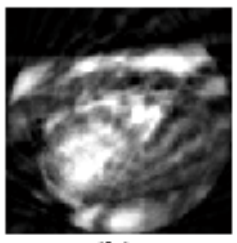

(b)

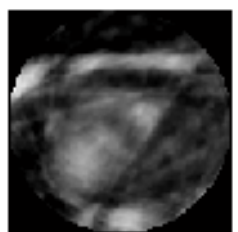

(c)

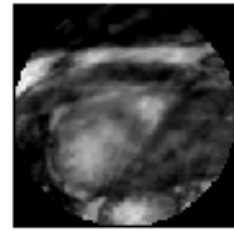

(d)

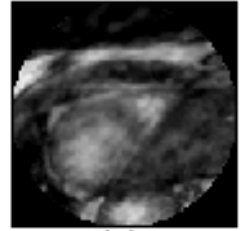

(e)

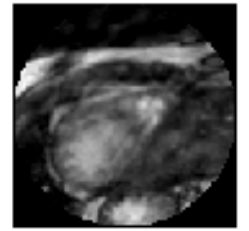

(f)

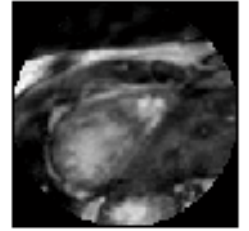

(g)

Figure 4. Cardiac image reconstruction at 10-fold acceleration. (a) Original image. (b) Image reconstructed using conventional gridding. Non-linear conjugate gradient reconstruction using (c) non-random radial $k$ - space, random radial $k$-space with $(\mathrm{d})$ variance $=0.5,(\mathrm{e})$ variance $=1,(\mathrm{f})$ variance $=2,(\mathrm{~g})$ variance $=3$. 